\title{
Implementing anti-racist pedagogy in health professional education: A realist review
}

\author{
Linda Diffey ${ }^{*}$ and Javier Mignone ${ }^{2}$ \\ ${ }^{1}$ Applied Health Sciences, University of Manitoba, Winnipeg, Manitoba, Canada \\ ${ }^{2}$ Department of Community Health Sciences, University of Manitoba, Winnipeg, Manitoba, Canada
}

\begin{abstract}
In response to the greater public awareness of anti-Indigenous racism within Canada's health care system, the nation's health education programs are under increasing pressure to incorporate anti-racist approaches in their training of health professionals. But implementation of curricular content about racism has been slow to emerge in these programs and a focus on culture rather than racial oppression persists. Few guidelines currently exist to assist in the application of anti-racist pedagogy in health education, and the published research in this area is relatively limited. The purpose of this systematic review was to explore the peer-reviewed literature using a critical realist framework to identify the factors and processes that influence the implementation of anti-racist teaching in a health context. The findings highlight the role of human actors, contextual factors, and pedagogical processes in either facilitating or impeding the advancement of anti-racist pedagogy in health education.
\end{abstract}

\section{Introduction}

Racism in the Canadian health care system has become increasingly recognized as an issue impacting indigenous peoples. Rooted in colonial history, anti-Indigenous racism permeates social institutions, including health care [1,2]. Stereotyping and other forms of discrimination can create environments in which Indigenous patients do not feel safe, contributing to systemic barriers that result in delayed diagnosis, treatment, and missed opportunities for preventive care [1]. It is imperative to attend to the underlying causes of mistrust, particularly among populations who are known to experience poorer health status than mainstream Canadian population [3,4] Incidents such as the 2008 death of an Indigenous man, untreated during the 34 hours he spent in the waiting room of a Canadian emergency department, underscore the dire need to examine how racial stereotyping operates in health care [5], prompting academics and physicians to make public calls for action around this issue [6,7].

Canadian professional organizations in the fields of nursing and medicine have codified the need to address anti-Indigenous racism. The Canadian Nurses Association [8] passed a resolution to improve health equity for Indigenous peoples through identifying strategies to reduce racism and structural discrimination. A policy statement from the Royal College of Physician and Surgeons of Canada [9] one year earlier stressed the importance of critical reflection and analysis by doctors to recognize the impacts of racism and oppression on Indigenous patients.

The inclusion of the topics of racism and colonial oppression within health professional training is gaining momentum. Frameworks for teaching Indigenous health in undergraduate medicine [10], post-graduate medicine [11], and baccalaureate nursing [12] have been developed through the collaborative efforts of Indigenous and non-Indigenous health professional organizations and academic associations. Central to these frameworks is the fostering of critical reflection skills that enable learners to identify and interrupt the processes that perpetuate colonial oppression in healthcare. The Truth and Reconciliation Commission of Canada [13], through its mandate to seek redress for the legacy of residential schools, included among its calls to action that Canadian medical and nursing schools institute a mandatory Indigenous health issues course that includes skills training in anti-racism.

Although addressing racism has been increasingly recognized as a critical subject area for the training of Canadian health professionals, very little has been published about how this is being implemented in the nation's professional health education programs. Implementation of curricular content about racism has been slow to emerge within Canadian schools of nursing and medicine, and teaching about Indigenous health has tended to focus on cultural awareness or competence and health disparities rather than concepts of colonial oppression, discrimination, power, and privilege [14-17]. Ly and Crowshoe [18] suggest that the lack of substantive training about racial stereotyping and the related social, political, and historical context not only place health professionals at a disadvantage in practice but also put Indigenous patients at risk.

Anti-racist pedagogy is an approach to teaching which seeks to identify, challenge and transform those aspects of a system that maintain, power, privilege and racism $[19,20]$. Race and social difference are explicitly named as issues of power and equity rather than cultural or ethnic matters [21]. Literature focusing on anti-racist pedagogical approaches in health education is limited [22], and has yet to emerge as a significant area of inquiry for Indigenous health

Correspondence to: Linda Diffey, Applied Health Sciences, University of Manitoba, Winnipeg, Manitoba, Canada, Linda Diffey, Tel: 204-975-7752; Fax: 204-975-7783; E-mail: Linda.Diffey@umanitoba.ca

Key words: anti-racist pedagogy, critical realism, health education, systematic review

Received: January 04, 2017; Accepted: January 18, 2017; Published: January 20, 2017 
education in Canadian professional health schools. The examination of the literature from non-health disciplines reveals the challenging nature of undertaking this approach in post-secondary education; resistance from students and administration, negative evaluations, and instructor discomfort and uncertainty are frequently described [23-26]. With few systematic examinations of how the implementation of antiracist education functions in health disciplines, there is little currently to guide professional health programs in Canada to address racism in the context of Indigenous peoples.

The purpose of this systematic review was to explore the experiences of health professional education programs to understand the process of implementing anti-racism initiatives. In particular, the review sought to identify the factors and mechanisms that may contribute to the feasibility and sustainability of employing an antiracist pedagogical approach in health professional education. For the purposes of this review, the World Health Organization definition of health professionals as those individuals who 'study, diagnose, treat and prevent human illness, injury and other physical and mental impairments in accordance with the needs of the populations they serve' [27] was used to shape the parameters of the literature search. Health professional education was operationalized as programs that train individuals to develop the knowledge, skills, attitudes, and behaviors required to earn or maintain the designation of health professional.

\section{Materials and methods}

\section{Critical realist approach}

The approach taken in this systematic review draws upon critical realism, which considers the social world as comprising multiple, interconnecting systems with complementary and countervailing mechanisms that interact in complex ways to influence outcomes [28]. Realism provides a particularly useful ontological framework for examining interventions in health education as outcomes are often highly context dependent and thus pose a challenge when reviewing the literature to gain insight into the best practices for implementation of particular teaching strategies [29]. Within the realm of anti-racist teaching interventions, critical realism offers a means to unpack the complexity of factors at play, including those related to power and oppression.

Consistent with realist review guidelines developed by Pawson, Wong and others, this review considers how outcomes emerge through their association to context and social processes or mechanisms [30,31]. Additionally, this review is also informed by the critical realist work of Houston [28] and Porter [32] who have argued for the consideration of human agency and time as distinct factors in describing causal chains: context + time + mechanism + human agency $=$ outcome Systematic review undertaken with a critical realist approach therefore requires the researcher to examine outcome patterns, social processes and structures, the social and temporal contexts, and the experiences and responses of the actors involved [28,32]. The intent of this review was not to evaluate whether teaching about racism is effective in professional health education, but rather to understand how antiracism teaching works when training health professionals, in what ways it is influenced by the social context, and the role of various actors (e.g. students, instructors, administrators) in this type of teaching.

\section{Inclusion and exclusion criteria}

Sources with rich descriptions of anti-racist educational programs or interventions for health professional training at any stage (i.e., undergraduate to post-graduate and continuing professional development) were reviewed. Documents were included if details about the educational intervention were described; the educational intervention was directed at students in health professional programs or practicing health professionals; and the intervention involved discussions about race, racism or racial oppression. Documents were excluded if the publication language was not English; the learners targeted for the education intervention were not in the health professions (e.g. pre-medicine, pre-nursing, patients or clients); or the teaching strategies described did not specifically address race, racism, or racial oppression. Opinion-driven articles, such as editorial reviews, commentaries, and those that did not describe a systematic process for examining interventions were excluded, as were articles outlining how guidelines were developed. Consistent with realist review guidelines, the defining of inclusion and exclusion criteria was iterative and refined during the process of identifying articles for the synthesis [30-32].

\section{Identification of articles}

Five electronic databases relevant to health education were searched from their inception dates to February 2015 using a search strategy developed by the authors in consultation with a health sciences librarian. Publications of any type were included, and the search was limited to English language documents. Authors' own reference libraries and the reference lists of studies found through the electronic database search were created. Details of the databases and search strategies are available in Supplemental File 1.

Screening of titles and abstracts against inclusion and exclusion criteria was conducted by the first author. Full text was obtained for 287 articles, which were rescreened for eligibility by the first author in consultation with the second author. Of the 94 eligible articles, 31 were deemed to have provided sufficient detail about the educational interventions to be included in the synthesis (Figure 1).

The 31 articles included in the systematic review ranged in publication dates from 1996 to 2015 and from a variety of disciplines, including social work (16), nursing (8), inter-professional/ multidisciplinary (4), medicine (1), public health (1), and midwifery (1). Most of the articles were from the USA (14) and Australia (5), and the remaining ones were from Canada (3), New Zealand (3), South Africa (2), UK (2), Scotland (1), and Israel (1). The articles also ranged in their focus, from examining specific learning activities (6), a module within a course (2), a whole course (10) or workshop (3), a complete curriculum (2), or the experience of instructors more generally (8). How these elements were examined also varied, from the analysis of student assignments (7), use of interviews or focus groups (7), survey methods (6), mixed methods (7), ethnography (1), evaluation (1), and quasi-experimental (1).

\section{Data extraction, organization, and analysis}

Building on the methods described by Rycroft-Malone, et al. [33], Kastner, et al. [34], and Wong, et al. [35], a data extraction form was designed. A critical appraisal section, based on the work of Ryan, et al. [36] was included in the form. A pre-test with three articles resulted in further refinement to the data extraction form (Supplemental File 2), which was then used to gather data on: (1) process details of the intervention, such as the level, focus, approach, target, and change agents; (2) context details, such as health discipline, setting, socioeconomic, and cultural factors; (3) moderating factors that influenced the outcome of the intervention; and (4) theories, either assumed or postulated, that formed the basis of the intervention. 


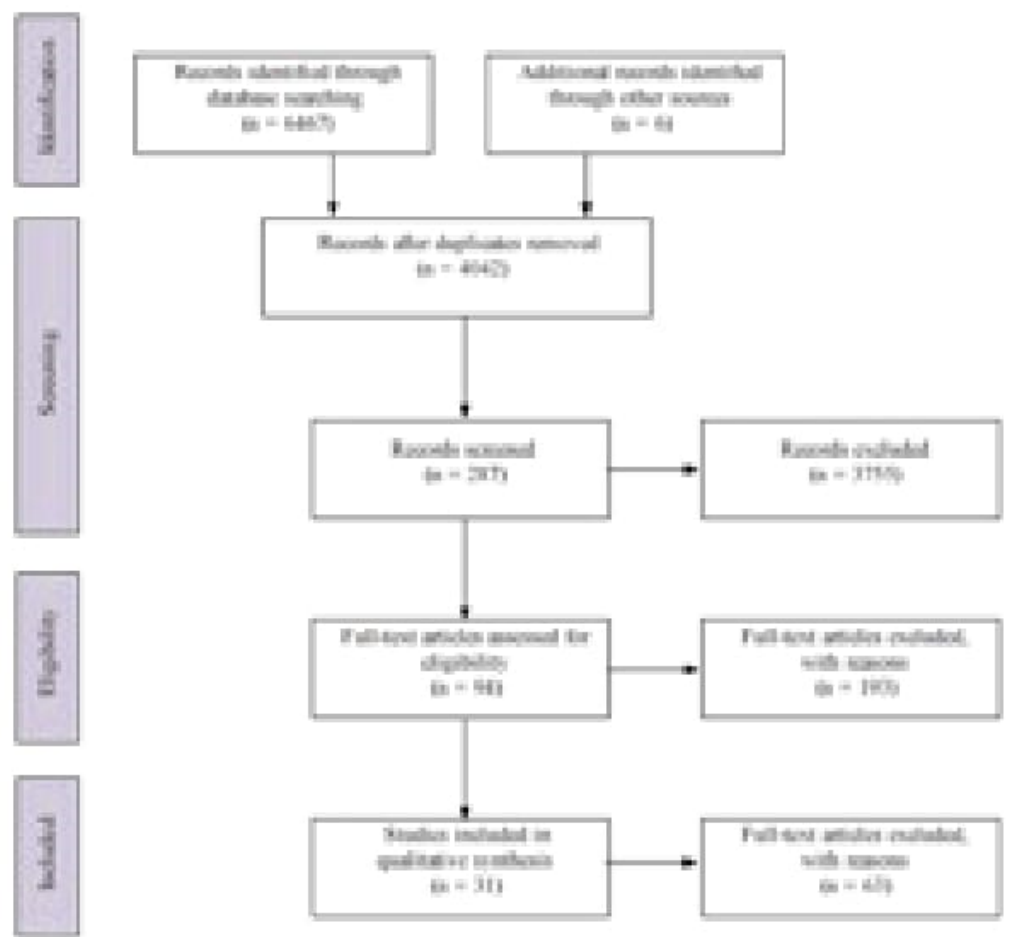

Figure 1. Flowchart outlining the process for locating and selecting the studies included in the systematic review. (Adapted from [72]).

Extracted data was then organized by interventions, factors, outcomes and appraisal to facilitate the identification of patterns in the data. Data extracted from the articles was further organized according to the emerging patterns related to context factors, process factors, agents of change, and temporal factors. Articles were read and re-read to understand the relationship of the identified factors and agents to the intervention outcomes.

\section{Results and discussion}

The findings were organized in four broad themes: pedagogical approaches; the role of actors; contextual factors; and pedagogical processes as mechanisms. Each of these themes was further specified and categorized.

\section{Pedagogical approaches}

The studies included in this review described a range of pedagogical approaches for incorporating race issues in the teaching of health professionals. These can be envisioned as occupying four categories defined by the primary focus of the pedagogical approach: dialogue across social groups; deconstructing power and privilege; student transformation; and application to practice.

\section{Dialogue across social groups}

Allen, et al. [37] and Drabble, et al. [38] described educational interventions that utilized a cross-cultural perspective. While this approach was found to increase students' understanding of cultural knowledge and respectful partnerships [38], it was less effective for understanding power, privilege and oppression [38] and in connecting the social determinants of health to racism [37]. Intergroup dialogue $[39,40]$ is an approach that gathers students into small, peer-facilitated groups based on social identity in order to foster cooperative learning and communication between the groups. Students were found to have increased cultural competency in knowledge, skills, and awareness domains [39]. In Arieli's study, students had to negotiate a number of challenges, such as feeling the need to be the 'ambassador' for their culture, feeling comfortable expressing their identity, and difficulties of discussing the dynamics of oppression [40]. A slightly different approach, black-white teaching partnership, was described by Gollan and O'Leary [41] in which instructors from different social identity groups are co-leaders in teaching, modeling accountability, awareness of whiteness, and critical self-reflection in the process. Students found this approach to be a positive experience that increased their awareness about concepts of whiteness, othering, and the realization that they did not understand the experience of Indigenous peoples as well as they once thought [41].

\section{Deconstructing power and privilege}

A number of studies looked at educational interventions that focused on how power and privilege operate in society to oppress racialized groups. The learning activities varied among the studies, but a common thread was the challenging of dominant worldviews and examination of system- and institution-level racism. Students in these interventions tended to react with strong emotional responses [42] including hostility, guilt, shame or feeling victimized and blamed [43]. Wariness about the subject matter [44] and reluctance to engage [45] were noted as being more pronounced at the beginning of the intervention and waned over time. Notable outcomes of this type of approach included improved critical thinking and reflexivity [45] and a demonstrated ability to identify the mechanisms of oppression and privilege that drive health inequities $[42,46]$. Although some students were found to devalue the teaching content [45], others could see the relevance to their practice [47]. 


\section{Student transformation}

Another category of interventions focused on guiding the student through a process of transformation, emphasizing the role of the student in this process. These approaches emphasize internal shifts within the student around their identity and core beliefs [48] or frames of reference for interpreting experiences [49]. Although instructors in one intervention assumed students would be able to translate their raised critical consciousness into practice, learners expressed a desire to be given concrete strategies and skills [50]. Signal, et al. [46] reported no consistent increase in students' self-reported advocacy following the intervention, but Deepak and Biggs [49] observed that students were able to mobilize their reactions into an anti-racist stance. Abrums and Leppa [51] found that the majority of students who demonstrated a deep integration of content and critical analysis were from underrepresented groups, but also noted that the relationship between social group membership and this type of learning is complex and would require investigation beyond the scope of their study.

\section{Practice-centered}

Translating learning into practice formed the focus of another category of educational interventions. This could take the form of hands-on experience, such as through community-based service learning experiences [52] or gaining practice in managing the challenges of classroom discussions surround race issues [53]. One approach employed modeling of behavior and principles by instructors to demonstrate the transfer of theory into practice [54], but students still reported a desire to learn about concrete tools for working with clients. Collins and Wilkie [55] examined the use of portfolio assignments as a means for assessing students' ability to apply theory in practice, but found that students struggled with providing in-depth analysis of structural or institutional racism in their practice learning experiences.

\section{The role of actors}

Change agents, either within or external to the learning institution, played a critical role in the educational interventions examined in this systematic review. Most of the studies focused on the role of actors within educational institutions, particularly the instructors and students engaged in anti-racism education interventions, and to a lesser degree institution administrator, other faculty, and those external to the institution.

\section{Instructors}

How instructors engaged in anti-racist educational interventions was influenced by a variety of factors, including their knowledge and expertise, their burden of responsibilities and access to support, and their social identity.

Instructors who were skilled in recognizing and responding to conflicts, potentially distressing opinions, and defensive responses among students helped to facilitate learning environments in which critical discussions emerged $[40,43,45]$. While few studies looked at the process of gaining experience in anti-racism teaching skills, Holland [56] found that the journey took place over several years, through a process of self-reflection that was gradual and punctuated with 'awakenings'. Self-reflection was also found to be valuable for developing anti-racist teaching practices in the setting of an experiential, hands-on learning workshop for faculty [53]. A number of studies described the use of an external expert to support instructors $[47,53,57]$. For courses that required multiple instructors, maintaining a cadre of teachers [58] and ensuring consistency [43] were challenges. Engagement of Aboriginal instructors in particular was found to be facilitated through the relationships and networks of a unit coordinator [43].

Some approaches to anti-racist teaching employed the social group identity of instructors as part of the pedagogical process. Students reported that co-facilitation of teaching by instructors from different groups offered access to multiple perspectives [41,43] and witnessing the modeling of a partnership in action [41]. For instructors from racially oppressed groups, teaching in this realm can involve considerable emotional labor as their identity is placed at risk [43], and they feel uncomfortable dealing with expectations from students of the same social group [59]. The lack of diversity among nursing faculty was identified as a barrier to implementing teaching that focuses on issues related to cultural diversity [60].

Many instructors were reluctant to teach about issues related to race or racism, and actively avoided these topics $[56,60]$. This discomfort was often attributed to a lack of adequate knowledge or training [56,60$62]$, the inherent difficult or unsettling nature of the topic $[59,60]$, or a feeling of lacking the credibility [56] or confidence [59] to tackle the subject. Forming a community of practice among like-minded instructors was identified as a facilitative process [57], whereas the lack of formal mentorship or a community of scholars was seen as a barrier to engaging in teaching about cultural safety [61].

\section{Students}

As with instructors, a variety of student-related factors that influenced the process of anti-racist teaching emerged. Young age and cultural isolation proved to be barriers to learning [56], but students with past experience in anti-racism training [55] or who had an interest in understanding how racism functions in their professional setting [47] were more receptive and more likely to engage in discussions about race and racism. Deepak and Biggs [49] found that referencing an event that impacted students or those close to them helped students to engage with the topic. Conversely, Beagan [58] found that exposing students to unfamiliar people and issues fostered learning.

Classroom dynamics were greatly influenced by students' social group identity. Having students from diverse social groups within a class could contribute to the challenging of racist views [37] and the creation of a learning environment that encourages dialogue [42]. However, students may also respond by self-segregating in order to create a comfort zone, which can be exclusionary for students in the minority [59]. In this context, moving students into mixed groups was met with initial resistance but ultimately had a positive impact on challenging stereotypes [59]. Intentionally dividing students by social group identity as a pedagogical strategy was found to foster an awareness of the pre-existing tensions between groups, but also led some students to feel silenced or like traitors if they did not agree with other members of their social group [40].

Students played an active role in their own learning about racism. Self-reflective writing provided a means for processing strong emotions and helping students to become more aware and enlightened about issues of privilege and oppression [42], although some students tended to focus on course concepts rather than deeper exploration of their own experiences $[63,64]$. Having students actively involved in the course design had variable outcomes. Creating ground rules for discussion as a group helped to ensure an atmosphere of emotional safety for student dialogue [53]. Requiring students to co-create the course outline led to considerable anxiety, and shed light on the paradox of students wanting to have input, yet also wanting an expert to give direction [54]. 
For instance, allowing a student to show a video clip in class created turmoil among students that lasted for weeks [40].

\section{Other institutional actors}

Institutional commitment to anti-racism through the inclusion of compulsory courses [43], and support among administrators and faculty to make teaching about oppression the norm [62] were identified as positive facilitating factors. Some instructors described resistance from management and colleagues about including racism-related topics [60], or pressure to focus on positive aspects of diversity and culture [56]. Others felt that the institution's responsibility to address racial discrimination was shifted onto lecturers without measures taken at organization-level policies and practices [59]. The burden of heavy academic workloads was seen as a factor in instructors' lack of energy to take on the challenge of anti-racism teaching [61]. Another source of tension was the absence of a shared understanding of how subjects such as race and culture should be approached [60].

\section{External actors}

The role of stakeholders outside academia was discussed in a few of the studies. Although community was identified as an important influence on curriculum, instructors noted the challenges of determining how they should be engaged and how to conceptualize 'community' [60]. Signal, et al. [46] noted the use of a needs assessment study to include the voice of stakeholders in curricular development, while [52] described the engagement of Native American community members in the planning of a community immersion learning experience. Involving outside agencies in off-site teaching was found to both facilitate and challenge the delivery of anti-racist teaching. For example, instructors in one study reported that agencies were more supportive than anticipated, yet students reported some degree of institutional resistance or lack of collaboration once engaged in an anti-racism field assignment within agencies [64]. Basham et al. [44], referencing the same context, noted some factors that moderated this process, including active engagement of the agency staff in planning, clarity of the goals and purpose of the students' placement and assignment, and providing the agency-based supervisor with sufficient information.

\section{Contextual factors}

A number of context-related factors were identified in the studies, and these ranged from the larger social environment to the climate within the educational institution and the more immediate setting of the classroom.

\section{Sociopolitical context}

Although the site of anti-racism teaching in health professional programs is typically the academic institution, the role of external contextual factors on the teaching process must also be considered. Racial tensions between groups in a region, including history of its development, are one such consideration. Arieli, et al. [40] observed an unequal power relationship between two groups of students in the classroom, which reflected the tension between Jews and Arabs in the larger population. Failure to consider the specific racial context leads to anti-racism education that does not adequately address community needs $[47,59]$. A shift in sociopolitical climate, as occurred in New Zealand with the government's placing priority on eliminating health inequalities among Maori people, was identified as a key factor in introducing anti-racism training [46,65]. Similarly, the policies and standards of professional regulating bodies were identified as significant drivers of anti-racism curricular implementation [45,49,55,60-62].

\section{Institutional climate}

While the influence of actors within institutions was discussed earlier, a number of other factors related to the institutional climate around anti-racist pedagogy emerged from the data. Barriers to implementing content about racial oppression included questioning the legitimacy of the content [58], hostility towards instructors [62], 'built in failure' through the hiring of unqualified instructors and the conveying of negative attitudes to students by other faculty members [62]. Power dynamics within the institution were also identified as influential factors. When power was viewed as being held by a select few at the top of an organizational structure, there was a perceived inability among instructors to be able to influence change and implement antiracist teaching approaches [47]. Curricular decisions were also seen as being influenced by the priorities of lobby groups and a desire to be 'politically correct', while also trying to avoid backlash [60].

The dominant perspectives operating in an institution can have a profound impact on the implementation of anti-racism approaches. A number of studies identified the dominance of the white perspective as problematic as it positions whiteness as normal and marginalizes the needs of ethnic minority students and stakeholders $[50,56,66]$. Dominance of 'culture' and 'diversity' in institutional discourse can act to keep racism and anti-racism in the background [56]. And the hegemonic culture of healthcare, which organizes curriculum around physiological systems and medical diagnoses, does not include parameters for including race in curricula [60].

The institution's readiness for change was identified as an important factor in the implementation of anti-racist teaching. Commitment from university administration to address racial oppression through the adoption of strategic plans fosters a supportive environment for instructors to incorporate anti-racist approaches in their teaching $[43,53,62]$. Taking the opportunity to introduce such a course during a time of larger curricular changes was described as a strategy, although resistance and a lack of respect within the institution in response to these courses was also noted [45,58].

In terms of context, most studies focused on the immediate learning environment of the student. Dynamics within the classroom influenced student engagement. Concerns about safety when sharing views [54], large class size $[40,56]$ or the dominance by several group members [45] were seen as barriers to dialogue and learning for students. Shifting the power dynamics towards student-led discussions resulted in vibrant dialogue, but also discomfort for the instructor related to the loss of control [54]. Student vulnerability, particularly for students of color, was a concern for instructors when trying to balance teaching about race and racism while not placing students in potentially dangerous situations $[44,56]$. While engaging in antiracism learning could provoke anxiety and discomfort for students, they also described feeling strengthened by the experience [57]. When expressions of racism manifest in the classroom, subsequent dialogue among students was challenging [37] and resulted in students adopting coping strategies to limit anxiety and stress [59]. Resistance by students to engage in discussions about racism was noted by a number of authors. For students who identify as part of the dominant culture, being challenged about their identity as members of the oppressive group may be expressed as negativity and resistance towards the instructor [65], indifference [62], denial [40,65], or reluctance to engage in self-examination [50]. Managing these challenging dynamics in the classroom can be difficult and frustrating for instructors [56,65] 
but can also provide motivation to find ways to convey the message [62] and make it a rewarding experience [65]. If efforts are made to incorporate disquiet as part of the learning process, it was seen as useful in transforming attitudes [45].

\section{Pedagogical processes as mechanisms}

The processes involved in any educational intervention are complex, and this proved to be the case in the anti-racist interventions considered in this review. Processes related to pedagogical planning and design, teaching methods, and assessment of students were the areas in which these mechanisms were situated.

The relationship between instructors' goals and students' expectations played a key role in learning process. Students may expect to focus on direct service issues around racism [66] and concrete strategies for managing race issues [50] whereas instructors may focus on systemic racism and view consciousness raising as the priority $[50,66]$. Being explicit about learning goals and how these apply to learning activities such as assignments emerged as a key factor in bridging this gap $[44,64]$.

Approaches to the pedagogical design process were variable among the studies in the review. For a number of instructors, finding a strategy to introduce race-issues in teaching was key, and these included starting with less threatening or divisive issues [40,53], utilizing small groups [53], and using discussions about privilege as a point of entry [50]. Despite such efforts to build trust among students before engaging in divisive issues, this did not necessarily eliminate the tension and discomfort associated with these discussions [40]. Frontloading a large volume of new, foundational concepts at the beginning of a course can be overwhelming for students, although this could be mediated through support from the instructor [54]. Beyond the introduction of race-related topics, students were found to struggle with the subject if there was a lack of sustained attention and the presentation of content was diffuse or disjointed [58]. Including opportunities for post-training follow up helped to establish an ongoing supportive community of instructors in one study involving a faculty development workshop [53], while Bozalek and Biersteker [57] found that undergraduate students did not access the available follow up support.

Using a variety of teaching materials [37], learning activities [41] and assignments [54] were all deemed as helpful by students. Incorporating videos that featured the experience of individuals from racially oppressed groups assisted in the facilitation of learning by large groups of students, particularly for community members who may not feel comfortable in large group settings [43]. Such videos helped to challenge stereotypes [43] and made a lasting impact that fostered empathy [49], although long and repetitive videos were viewed as less effective [43]. Instructor-facilitated discussion before and after viewing videos helped students to translate their emotional responses into an anti-racist stance [49]. Case-based studies helped to focus information for students [46] and prompted discussion [37].

In-person discussions among students were found to help students to raise emotionally difficult topics $[37,49]$. Blended courses that supplemented classroom meetings with online discussions required sufficient numbers of well-trained facilitators to respond to students' emotional distress in the web-based forums [57]. Students who remained anonymous during online discussions were less likely to engage in higher risk dialogues [57]. Visual exercises that involved drawing were found to be a stimulus for group interaction that in turn fostered insights and learning among students [57].
Instructors' modeling of teaching principles was a deliberate pedagogical tool described in a number of the studies, and this took a variety of forms. Modeling of intercultural teaching partnerships provided an opportunity to deconstruct how the partnership worked and how to respond to challenges, skills that were seen as helpful for real world practice [41]. Kickett, et al. [43] found that such co-coordination required strong leadership skills and high emotional intelligence. Another opportunity for modeling intercultural communication was in the setting of community-based learning in which the instructor is required to shift into the role of guest or visitor, although this can be challenging in terms of letting go of control over the learning process [52]. Modeling of social justice principles through transparency of teaching processes and use of self-disclosure positions the instructor in a vulnerable place and tended to be more useful for learning by students who possessed considerable practice experience prior to the course [54].

Most of the studies in this review did not examine the processes used by instructors to assess students' learning of anti-racism content. Of those that did, the process of self-reflection featured prominently. Students who were asked to reflect on their own self-interview from the beginning of the course had to engage in considerable emotional processing but demonstrated greater awareness of their own privilege or oppression [42]. Narrative analysis of nursing students' final reflective papers revealed varying levels of analysis and synthesis of course content on race, class, and privilege, although the majority of students who demonstrated the highest level of learning were from non-dominant social backgrounds [67]. Both Donner, et al. [64] and Collins and Wilkie [55] examined social work students' portfolio reflections on an anti-racism field placement project, and found that in-depth analysis of systemic oppression and examination of personal learning were lacking to a large degree.

\section{Conclusion}

The study had several limitations. Language bias is an important one, given that the literature reviewed was only in English, consequently missing out on potentially valuable studies in a variety of different countries and regions that seek to include anti-racism pedagogies. Specific to the main motivation of this study, there were few studies that dealt with anti-racism education programs more specifically related to Indigenous populations. Finally, the variety of programs reviewed, while offering a rich set of differing experiences, made their comparability more difficult.

The study systematically explored the peer-reviewed literature on experiences of health professional education programs that have incorporated curricular content on the topic of racism. The objective was to understand how and under what circumstances this pedagogical approach may be successfully implemented. It also sought to understand the process of implementing anti-racism initiatives in health professional education programs, and to identify the factors and mechanisms that contribute to the feasibility and sustainability of employing these anti-racist pedagogical approaches. The findings were organized in four broad themes: pedagogical approaches; the role of actors; contextual factors; and pedagogical processes as mechanisms. Each of these themes was further specified and categorized.

Four categories of pedagogical approaches for incorporating race issues in the teaching of health professionals, defined by their primary focus, were identified: dialogue across social groups, deconstructing power and privilege; student transformation; and application to practice. Each showed strengths and weaknesses. For instance, 
dialogue across groups helped increase students' understanding of cultural knowledge, but less so of power. Deconstructing power and privilege helped improve critical thinking and reflexivity and to identify mechanisms of oppression, although frequently it was met with strong emotional responses of hostility, guilt, and feeling victimized. The student transformation approach emphasized the role of the student, which assisted in deep integration of content and critical analysis. However, learners expressed the desire for more concrete strategies and skills. The practice-centered approach facilitated hands-on experience, but students struggled with providing in-depth analysis of structural or institutional racism in their learning experiences.

The review also examined the role of actors as change agents, be they instructors, students, other institutional actors, or external actors. Instructors were influenced in their engagement in anti-racist educational interventions by factors such as knowledge and expertise, burden of responsibilities, access to support, and social identity. In relation to students, a variety of factors influenced the process of antiracist teaching, among them cultural isolation, interest in understanding racism, social group identity, classroom dynamics, and particular techniques such as self-reflective writing, ground rules for discussion, and involvement in course design. Key among institutional actors was institutional commitment to anti-racism through the inclusion of compulsory courses and the support among administrators. The role of external stakeholders, although less discussed, was found to both facilitate and challenge the delivery of anti-racist teaching.

Among contextual factors, the sociopolitical context and the institutional climate emerged as central aspects. In relation to the latter, racial tensions between groups in a region, including the history of its development, was a key consideration. The institutional climate involved a number of components, such as institutional commitment, power dynamics, and dominant perspectives operating in an institution. Some aspects were identified as problematic, among them the dominance of the white perspective, 'culture' and 'diversity' in the institutional discourse acting to keep racism in the background, and hegemonic culture of healthcare which organizes curriculum around physiological systems and medical diagnoses.

The approaches to pedagogical design varied significantly among the studies reviewed, providing a range of creative options. However, most studies did not examine the processes used to assess students' learning of anti-racism content.

The review coincides with earlier literature that describes antiracist pedagogy as a framework that is intended to transform systems that uphold the mechanisms of racial oppression [19,21,25], while suggesting numerous limitations toward achieving this goal. Among the inconsistencies was how the review showed programs where race and social difference are dealt with explicitly as they relate to power and equity, albeit other instances where they were considered as cultural or ethnic matters. Collapsing issues such as poverty, racism, and violence into the less threatening concept of culture effectively conceals these issues from critical examination $[68,69]$. The review confirmed what other authors have argued, that anti-racist pedagogical approaches in health education are somewhat limited [22]. The literature related to professional health programs in Canada to address racism in the context of Indigenous peoples was almost non-existent.

This review was informed by the critical realist work of Houston [28] and Porter [32] as a means of describing the role of context, time, mechanisms, and human agency in anti-racist health education and how these factors influence program outcomes. Central to this process is the development of a program theory or theory of change, which provides an understanding of why a class of intervention is thought to 'work' in generating an outcome [30,31,70,71]. The literature reviewed did not provide substantive evidence in relation to the program theories of change of the different initiatives. The study has however provided some initial elements that merit further investigation that may lead to a detailed formulation of a program theory of change. These relate to context factors that may enhance or hinder the implementation of antiracist education programs; factors related to actors and agency; and issues of institutional readiness and time factors.

\section{Authorship and contributorship}

The authors of this manuscript have both contributed substantially to the conception, design, data acquisition and analysis as well as the drafting and revising of the manuscript.

\section{Acknowledgements}

The authors would like to acknowledge Janice Linton at the Neil John Maclean Health Sciences Library, University of Manitoba for her advice on the search strategy used in this review.

\section{Funding information}

There was no funding source associated with the research reported in this manuscript.

\section{Competing interest}

The authors declare that they have no competing interests.

\section{Disclosure statement}

The authors have no financial or benefits related to this research to disclose.

\section{References}

1. Health Council of Canada, Empathy, dignity, and respect: Creating cultural safety for Aboriginal people in urban health care (Health Council of Canada, Toronto, 2012).

2. Allan B, Smylie J (2015) First peoples, second class treatment: The role of racism in the health and well-being of Indigenous people in Canada.

3. Manitoba Aboriginal and Northern Affairs, Aboriginal people in Manitoba. (Manitoba Department of Aboriginal and Northern Affairs, Winnipeg, MB, 2012).

4. Reading C, Wien F (2009) Health Inequalities and Social Determinants of Aboriginal Peoples Health (National Collaborating Centre for Aboriginal Health, Prince George, BC, 2009).

5. Hart MA, Lavallee B (2015) The Social Determinants of Health in Manitoba, L Fernandez, S. MacKinnon, J. Silver, Eds. (Canadian Centre for Policy Alternatives, Winnipeg, MB, 2015) 10: 145-160.

6. Browne AJ (2014) Racism in health system: Expert Working Group gets at factor sidelined at Sinclair inquest.

7. Elkins D (2009) Investigate "institutional racism" in healthcare: Indigenous MDs.

8. Canadian Nurses Association (2014) Addressing Racism and Discrimination to Improve Health Equity for Aboriginal Peoples. Ottawa, ON: Canadian Nurses Association.

9. The Indigenous Health Advisory Committee, The Office of health Policy and Communications, 2013. Indigenous health values and principles statement. Ottawa, ON: Royal College of Physicians and Surgeons of Canada.

10. Lavallee B, Neville A, Anderson M, Shore B, Diffey L (2009) Eds., First Nations, Metis, Inuit Health Core Competencies: A curriculum framework for undergraduate medical education, (Indigenous Physicians Association of Canada and the Association of Faculties of Medicine of Canada, Canada, 2009).

11. Anderson M (2009) Eds., First Nations, Metis, Inuit Health Core Competencies: A curriculum framework for continuing medical education, (Indigenous Physicians Association of Canada and the Royal College of Physicians and Surgeons of Canada, 2009). 
12. Hart-Wasekeesikaw F (2009) Cultural competence and cultural safety in nursing education: A framework for First Nations, Inuit and Metis nursing, (Aboriginal Nurses Association of Canada, Ottawa, ON, 2009).

13. Truth and Reconciliation Commission of Canada, Truth and Reconciliation Commission of Canada: Calls to Action, (Winnipeg, 2015).

14. Azad N, Power B, Dollin J, Chery S (2002) Cultural sensitivity training in Canadian medical schools. Acad Med 77: 222-228. [Crossref]

15. Redwood-Campbell L, MacDonald WA, Moore K (1999) Residents' exposure to aboriginal health issues. Survey of family medicine programs in Canada. Canadian Family Physician 45: 325-330.

16. Rowan MS, Rukholm E, Bourque-Bearskin L, Baker C, Voyageur E, et al. (2013) Cultural competence and cultural safety in Canadian schools of nursing: a mixed methods study. Int J Nurs Educ Scholarsh 10. [Crossref]

17. Saylor K (2012) Development of a curriculum on the health of Aboriginal children in Canada. Paediatr Child Health 17: 365-367. [Crossref]

18. Ly A, Crowshoe L (2015) Stereotypes are reality': addressing stereotyping in Canadian Aboriginal medical education. Med Educ 49: 612-622. [Crossref]

19. McGibbon E, Etowa J (2009) Anti-racist health care practice. (Canadian Scholars' Press, Toronto, ON, 2009).

20. Schroeder C, DiAngelo R (2010) Addressing whiteness in nursing education: The sociopolitical climate project at the University Of Washington School of nursing. $A d v$ Nurs Sci 33: 244-255.

21. Dei GJS (1996) Anti-racism education: theory and practice. (Fernwood Publishing, Halifax, 1996).

22. Hassouneh D (2009) Anti-racist pedagogy: Challenges faced by faculty of color in predominantly white schools of nursing. J Nurs Educ 45: 255-262.

23. Peden S (2011) Dancing with the elephant: teacher education for the inclusion of First Nations, Me'tis and Inuit histories, worldviews and pedagogies. (University of Manitoba, Winnipeg, MB, 2011)

24. Boatright-Horowitz SL, Marraccini M, Harps-Logan Y (2012) Teaching Antiracism: College Students' Emotional and Cognitive Reactions to Learning About White Privilege. J Black Stud 43: 893-911.

25. DiAngelo R, Flynn D (2010) Showing what we tell: Facilitating antiracist education in cross-racial teams. Understanding and Dismantling Privilege 1: 1-24.

26. DiAngelo R, Sensoy O (2014) Getting Slammed: White Depictions of Race Discussions as Arenas of Violence. Race, Ethnicity and Education 17: 103-128.

27. World Health Organization (2013) Transforming and scaling up health professionals' education and training: World Health Organization guidelines 2013, (Geneva, Switzerland, 2013).

28. Houston S (2010) Prising Open the Black Box: Critical Realism, Action Research and Social Work. Qual Soc Work 9: 73-91.

29. Wong G, Greenhalgh T, Westhorp G, Pawson R (2012) Realist methods in medical education research: what are they and what can they contribute? Med Educ 46: 89-96. [Crossref]

30. Pawson R, Greenhalgh T, Harvey G, Walshe K (2005) Realist review--a new method of systematic review designed for complex policy interventions. J Health Serv Res Policy 10 Suppl 1: 21-34. [Crossref]

31. Wong G, Greenhalgh T, Westhorp G, Buckingham J, Pawson R (2013) RAMESES publication standards: realist syntheses. BMC Medicine 11: 21.

32. Porter $\mathrm{S}$ (2015) The uncritical realism of realist evaluation. Evaluation 21: 65-82.

33. Rycroft-Malone J, McCormack B, Hutchinson AM, DeCorby K, Bucknall TK, et al. (2012) Realist synthesis: illustrating the method for implementation research. Implement Sci 7: 33. [Crossref]

34. Kastner M, Makarski J, Hayden L, Durocher L, Chatterjee A, et al. (2013) Making sense of complex data: a mapping process for analyzing findings of a realist review on guideline implementability. BMC Med Res Methodol 13: 112. [Crossref]

35. Wong G, Greenhalgh T, Pawson R (2010) Internet-based medical education: a realist review of what works, for whom and in what circumstances. BMC Med Educ 10: 12.

36. Ryan F, Coughlan M, Cronin P (2007) Step-by-step guide to critiquing research. Part 2: Qualitative research. BrJ Nurs 16: 738-744. [Crossref]

37. Allen J, Brown L, Duff C, Nesbitt P, Hepner A (2013) Development and evaluation of a teaching and learning approach in cross-cultural care and antidiscrimination in university nursing students. Nurse Education Today 33: 1592-1598.

38. Drabble L, Sen S, Oppenheimer SY (2012) Integrating a Transcultural Perspective into the Social Work Curriculum: A Descriptive and Exploratory Study. J Teach Soc Work 32: 204-221.

39. Humphreys ML (2012) Intergroup dialogue: A pedagogical model for integrating cultural competence within a social justice framework. Int J Interdiscip Soc Sci 6: 199213

40. Arieli D, Friedman VJ, Hirschfeld MJ (2012) Challenges on the path to cultural safety in nursing education. Int Nurs Rev 59: 187-193. [Crossref]

41. Gollan S, O'Leary PJ (2009) Teaching culturally competent social work practice through black and white pedagogical partnerships. Soc Work Educ 28: 707-721.

42. Garcia B, Van Soest D (1997) Changing Perceptions of Diversity and Oppression: MSW Students Discuss the Effects of a Required Course. J Soc Work Educ 33: 119-129.

43. Kickett M, Hoffman J, Flavell H (2014) A model for large-scale, interprofessional, compulsory cross-cultural education with an indigenous focus. J Allied Health 43: 38 44.

44. Basham KK, Donner S, Everett J (2001) A Controversial Commitment: The AntiRacism Field Assignment. J Teach Soc Work 21: 157-174.

45. Thackrah RD, Thompson SC (2013) Confronting uncomfortable truths: receptivity and resistance to Aboriginal content in midwifery education. Contemp Nurse 46: 113-122.

46. Signal L, Martin J, Reid P, Carroll C, Howden-Chapman P, et al. (2007) Tackling health inequalities: moving theory to action. Int $J$ Equity Health 6: 12. [Crossref]

47. Havens BE, Yonas MA, Mason MA, Eng E, Farrar VD (2011) Eliminating inequities in health care: understanding perceptions and participation in an antiracism initiative. Health Promot Pract 12: 848-857.

48. Van Soest D (1996) Impact of Social Work Education on Student Attitudes and Behavior concerning Oppression. J Soc Work Educ 32: 191-202.

49. Deepak A, Biggs MJG (2011) Intimate Technology: A Tool for Teaching Anti-Racism in Social Work Education. Journal of Ethnic and Cultural Diversity in Social Work 20: $39-56$.

50. Jeffery D (2005) What good is anti-racist social work if you can't master it?: Exploring a paradox in anti-racist social work education. Race Ethn Educ 8: 409-425.

51. Abrums ME, Leppa C (2001) Beyond cultural competence: teaching about race, gender, class, and sexual orientation. J Nurs Educ 40: 270-275. [Crossref]

52. Bolea PS (2012) Cross-Cultural Service Learning with Native Americans: Pedagogy for Building Cultural Competence. J Teach Soc Work 32: 284-299.

53. Van Jaarsveldt DE (2013) in Proceedings of teh 2013 IEEE 63rd Annual Conference International Council for Educational Media. (International Council for Educatio Meida, 2013)

54. Campbell C, Scott-Lincourt R, Brennan K (2010) The real world of the ivory tower: Linking classroom and practice via pedagogical modeling. J Teach Soc Work 28: 35-51.

55. Collins S, Wilkie L (2010) Anti-oppressive practice and social work students' portfolios in Scotland. Soc Work Educ 29: 760-777.

56. Holland AE (2015) The lived experience of teaching about race in cultural nursing education. J Transcult Nurs 26: 92-100. [Crossref]

57. Bozalek V, Biersteker L (2010) Exploring power and privilege using participatory learning and action techniques. Soc Work Educ 29: 551-572.

58. Beagan BL (2003) Teaching social and cultural awareness to medical students: "It's all very nice to talk about it in theory, but ultimately it makes no difference". Acad Med 78: 605-614.

59. Tilki M (2007) Racism: the implications for nursing education. Divers Heal Soc Care 4: 303-312.

60. Parker V, McMillan M (2008) Beyond barriers. The challenge of cultural diversity for nurse academics in the Australian context. Collegian 15: 143-149. [Crossref]

61. Wepa D (2003) An exploration of the experiences of cultural safety educators in New Zealand: an action research approach. $J$ Transcult Nurs 14: 339-348. [Crossref]

62. Singleton SM (1994) Faculty Personal Comfort and the Teaching of Content on Racia Oppression.

63. Littlefield MB, Bertera EM (2004) A Discourse Analysis of Online Dialogs in Social 
Diffey L (2017) Implementing anti-racist pedagogy in health professional education: A realist review

Work Diversity Courses: Topical Themes, Depth, and Tone. J Teach Soc Work 24: 16.

64. Donner S, Everett J, Basham K (2004) The Antiracism Field Assignment: Beginning Results and Lessons Learned. J Teach Soc Work 24: 17.

65. Richardson F, Carryer J (2005) Teaching cultural safety in a New Zealand nursing education program. J Nurs Educ 44: 201-208. [Crossref]

66. Williams C, Parrott L (2014) Anti-racism and predominantly 'White areas': Local and national referents in the search for race equality in social work education. $\mathrm{Br} J \mathrm{Soc}$ Work 44: 290-309.

67. Abrums ME, Resnick J, Irving LL (2010) Journey or destination? Evaluating student learning about race, class, and privilege in health care. Nurs Educ Perspect 31: 160166. [Crossref]
68. Gregg J, Saha S (2006) Losing culture on the way to competence: the use and misuse of culture in medical education. Acad Med 81: 542-547. [Crossref]

69. Schick C, St. Denis V (2005) Troubling National Discourses in Anti-Racist Curricular Planning. Can J Educ 28: 295-317.

70. De Silva MJ (2014) Theory of Change: a theory-driven approach to enhance the Medical Research Council's framework for complex interventions. Trials 15: 267.

71. Brennan N, Bryce M, Pearson M, Wong G, Cooper C, et al. (2014) Understanding how appraisal of doctors produces its effects: a realist review protocol. BMJ Open 4: e005466. [Crossref]

72. Moher D, Liberati A, Tetzlaff J, Altman DG (2009) Preferred reporting items for systematic reviews and meta-analyses: the PRISMA statement.

Copyright: (2017 Diffey L. This is an open-access article distributed under the terms of the Creative Commons Attribution License, which permits unrestricted use, distribution, and reproduction in any medium, provided the original author and source are credited. 